\title{
Multi-wavelength VLA and Spacecraft Observations of Evolving Coronal Structures Outside Flares
}

\author{
R.F. Willson \\ Department of Physics and Astronomy, Tufts University, Medford, MA 02155, USA
}

\begin{abstract}
Multi-wavelength Very Large Array (VLA), SOHO and RHESSI observations have been used to study the three- dimensional structure of evolving coronal loops and related signatures of impulsive and long-lasting energy release above active regions. Here, we discuss recent multiwavelength observations of an impulsive hard X-ray burst that was preceded by gradual changes in the structure of an extended coronal loop connecting the active region to one located on the other side of the solar equator, suggesting pre-burst magnetic evolution at different spatial scales. Changes in the 6 and $20 \mathrm{~cm}$ brightness temperature were also detected from the opposite footpoint region suggesting some sort of magnetic connectvity across the solar equator.
\end{abstract}

\section{Introduction}

Soft X-ray and Extreme Ultraviolet (EUV) images from the Yohkoh, SOHO and TRACE solar missions have been used to study the complex distribution of loops that outlines the large-scale structure of the solar corona within and outside of active regions. These global structures are thought to evolve in response to changes in the underlying magnetic fields or to currents and particle acceleration in the corona.

Radio observations with the Very Large Array (VLA) can extend the results of these orbiting solar missions by providing complementary information on the size, location, and temporal evolution of coronal loop structures where magnetic energy is stored and released. At 6 and $20 \mathrm{~cm}$ wavelength, the VLA can image slowly- varying coronal loops whose emission is produced by the thermal bremsstrahlung or thermal gyroresonance radiation of hot coronal plasma trapped in closed magnetic loops anchored to underlying sunspots. Full-disk VLA observations at $91 \mathrm{~cm}$ wavelength have also revealed large-scale coronal features that can connect distant regions on the Sun (Lang, Willson and Trottet 1988) and VLA observations of simultaneous burst activity at $20 \mathrm{~cm}$ wavelength have provided evidence for magnetic loops that can confine and channel energetic particles over large distances (Willson, Lang and Gary 1993).

Here, we discuss recent multi-wavelength observations that combine VLA snapshot maps at 6 and $20 \mathrm{~cm}$ wavelength with SOHO EIT and RHESSI data at EUV and hard X-ray wavelengths. VLA maps at 6 and 20 have been used to study the radio counterparts of evolving EUV loops and impulsive hard X-ray burst sources within two closely-spaced active regions on oposite sides of the solar equator. The combined results have allowed us to study evolving coronal sources at different heights and to investigate the effect that energy release in one part of an active region may have had on the structure of the same or distant active region. 

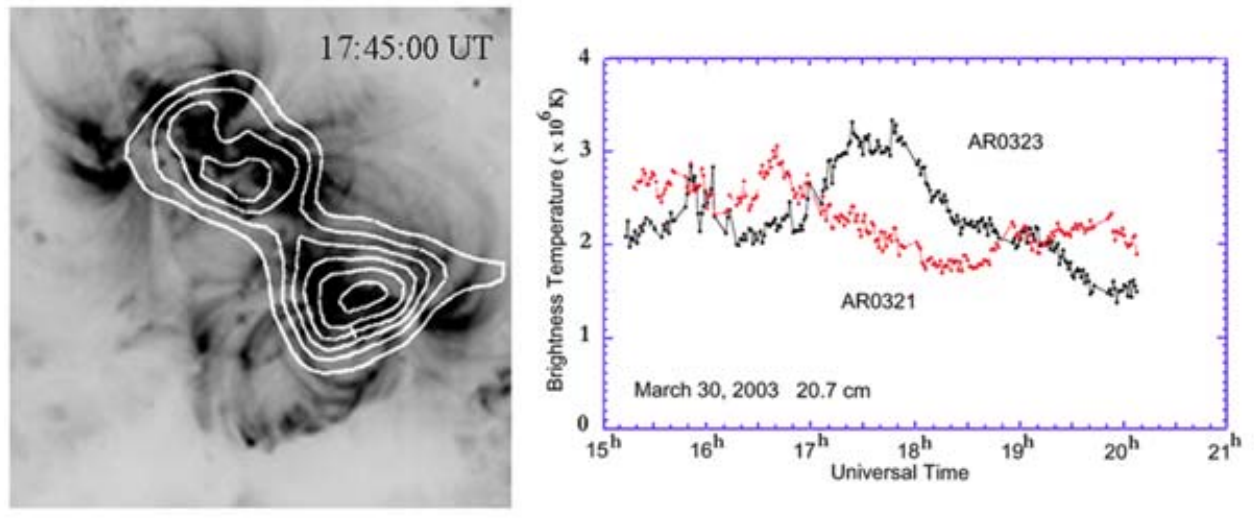

Figure 1. (left)VLA snapshot maps at 20.7 and $6.2 \mathrm{~cm}$ (inset) wavelength at $17: 36$ UT are overlaid on a SOHO MDI photospheric magnetogram taken at about the same time. (right) A plot of the peak $20.7 \mathrm{~cm}$ brightness temperature from active regions AR0321 and AR0323.

\section{Observations on 30 March 2003}

On 2003 March 30, the VLA (C configuration) detected bright $\left(\mathrm{T}_{b}=2-3.2 \times 10^{6} \mathrm{~K}\right.$ ), unpolarized $\left(\rho_{c} \leqslant 10 \%\right)$ emission at $20 \mathrm{~cm}$ wavelength from active regions AR0321 (N04 E11) and AR0323 (S08 W01) and from the trans-equatorial region between them (Fig. 3). Bright emission at $6.2 \mathrm{~cm}$ wavelength from the target region AR0323 was also detected; reliable images at $6.2 \mathrm{~cm}$ were not obtained for AR0321, located 5' to the northeast, because this active region was outside of the half-power point of the VLA primary beam.

VLA snapshot maps (1-minute intervals) at $20.7 \mathrm{~cm}$ indicate that these sources underwent significant changes in brightness temperature over the five hour period of observation. As illustrated in Figure 1, the peak brightness temperature of AR0323 began to increase from $\mathrm{T}_{b} \approx 2 \times 10^{6} \mathrm{~K}$ at $\approx 17: 00 \mathrm{UT}$ and reached a maximum of $\mathrm{T}_{b}=3.2 \times 10^{6}$ $\mathrm{K}$ about an hour later. It then declined steadily to about $\mathrm{T}_{b}=1.5 \times 10^{6} \mathrm{~K}$ by 20:00 UT. In contrast, the peak brightness temperature of AR0321 began to decline steadily from a peak of $\mathrm{T}_{b}=2.7 \times 10^{6} \mathrm{~K}$ at $\approx 16: 40 \mathrm{UT}$ to $\mathrm{T}_{b}=1.8 \times 10^{6} \mathrm{~K}$ at 18:30 UT followed by a small increase over the next hour.

These $20 \mathrm{~cm}$ wavelength variations were accompanied by hard X-ray burst emission from AR0321 (RHESSI) and by changes in the structure of the Fe XII $195 \AA$ EUV loop emission (SOHO EIT) in the equatorial region between the two active regions. As shown in Figure 2, a relatively weak "precursor" burst was detected by RHESSI at 17:35 UT, followed by a significantly larger event at 17:44 UT. The main hard X-ray burst was followed a C1.3-class GOES soft X-ray burst at 17:47 UT. RHESSI snapshot maps in the energy range between 6-12 keV (Fig 2) indicate that the burst and its precursor originated at the same location within AR0321. The impulsive hard X-ray bursts were then followed by more slowly-varying changes in the EUV emission detected in the transequatorial region between AR0321 and AR0323. As can be seen in the series of EIT images shown in Figure 3, the diamond-shaped area of low-brightness EUV emission along the southeastern edge of AR0321 began to extend westward along the solar equator starting at $\approx 18: 00$ UT. This evolving EUV source probably delineates plasma at a higher or lower temperature than that outlined by the Fe XII emission which has a peak formation temperature of $\mathrm{T} \approx 1.5 \times 10^{6} \mathrm{~K}$. This moving plasma might then be attributed to flare ejecta from the hard X-ray burst or to heating of the coronal loops in this region. We note that the intensity of the $20 \mathrm{~cm}$ loop in the trans-equatorial region overlying the 

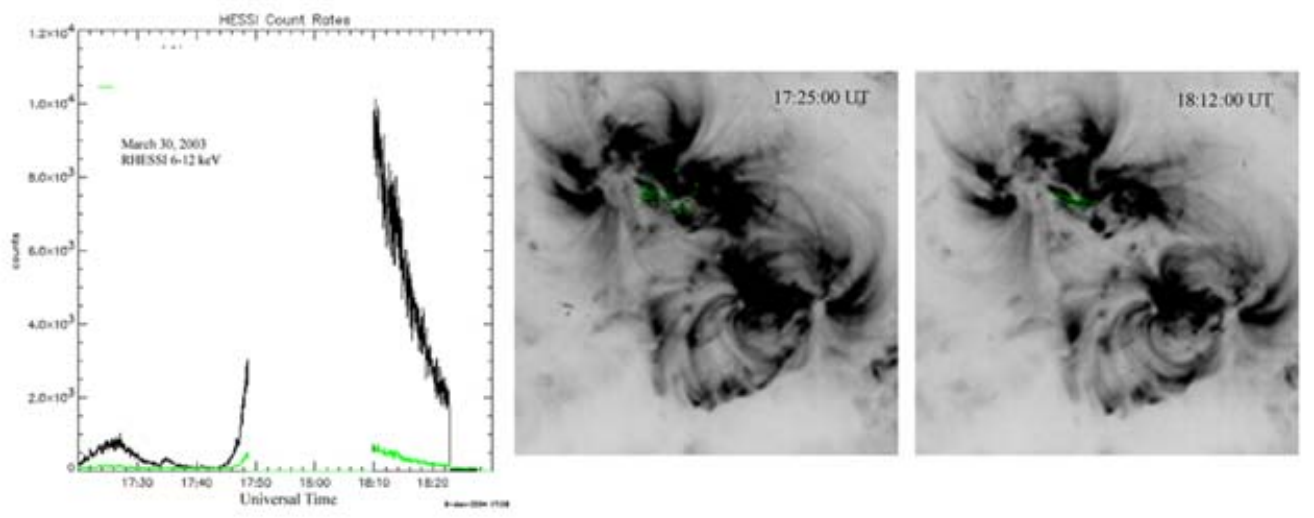

Figure 2. (left) Time profile of the RHESSI count rate in the energy range between 6.2 $9.2 \mathrm{keV}$ and $9.2-12.3 \mathrm{keV}$ showing the hard X-ray burst emission between 17:25-18:25 UT. (right) RHESSI snapshot images (20 sec intervals) are overlaid on SOHO EIT $195 \AA$ images of AR0321 at the times indicated. Here, the field-of-view is $10^{\prime}$ x $10^{\prime}$

evolving EUV emission decreased in intensity during this period, probably in response to the changing electron temperature, density, or both.

As shown in Figure 3, the $6 \mathrm{~cm}$ emission from AR0323 also varied significantly during this time period. The $6 \mathrm{~cm}$ emission consists of two main sources ( $\mathrm{W}$ and $\mathrm{E}$ ) with a fainter bridge of emission connecting them. These sources overly the leading and trailing sunspots of negative and positive magnetic polarity, respectively, while the narrower bridge overlies a more compact area of predominantly negative polarity near the center of the active region. These evolving $6 \mathrm{~cm}$ sources are most-likely attributed to thermal gyroresonance emission at the second or third harmonic, s, of the gyrofrequency; they therefore outline coronal plasma where the magnetic field strength has a value of $\mathrm{B}=$ $857 \mathrm{G}(\mathrm{s}=2)$ or $\mathrm{B}=570 \mathrm{G}(\mathrm{s}=3)$. Examination of a series of MDI magnetograms (Fig 2.) show the growth and migration of a number of small magnetic elements (arrows) between the sunspots during the period of the VLA observations Snapshot maps at both 6 and $20 \mathrm{~cm}$ wavelength as well as images from RHESSI showed no evidence for low-level impulsive bursts from AR0323 as might be expected if these evolving magnetic fields induced electric currents and triggered magnetic reconnection events and the production of energetic particles whose impulsive gyrosynchrotron emission could be detected at radio wavelengths. We therefore suspect that the changes in brightness temperature shown in Figures 1 and 4 are related to slower resistive heating of the coronal plasma in response to these evolving photospheric magnetic fields.

\section{Summary and Conclusions}

Our multiwavelength VLA, SOHO and RHESSI observations have shown that the complex coronal environment above active regions may be influenced by energy release or magnetic reconfiguration initiated outside the active regions. On 30 March 2003, slowlyevolving $20 \mathrm{~cm}$ emission associated with coronal loops joining sunspots of opposite magnetic polarity in the same active region as well as in large-scale trans-equatorial loops joining the two regions was observed during times of impulsive and slow energy release. The evolving $20 \mathrm{~cm}$ loops may have been part of a more extensive magnetic reconfiguration involving impulsive hard X-ray bursts in one region and slow magnetic heating in the other. As described by Uchida et al (1992) gradual expansion of anti-parallel magnetic fields in the two active regions may drive magnetic reconnection and supply flux to a 

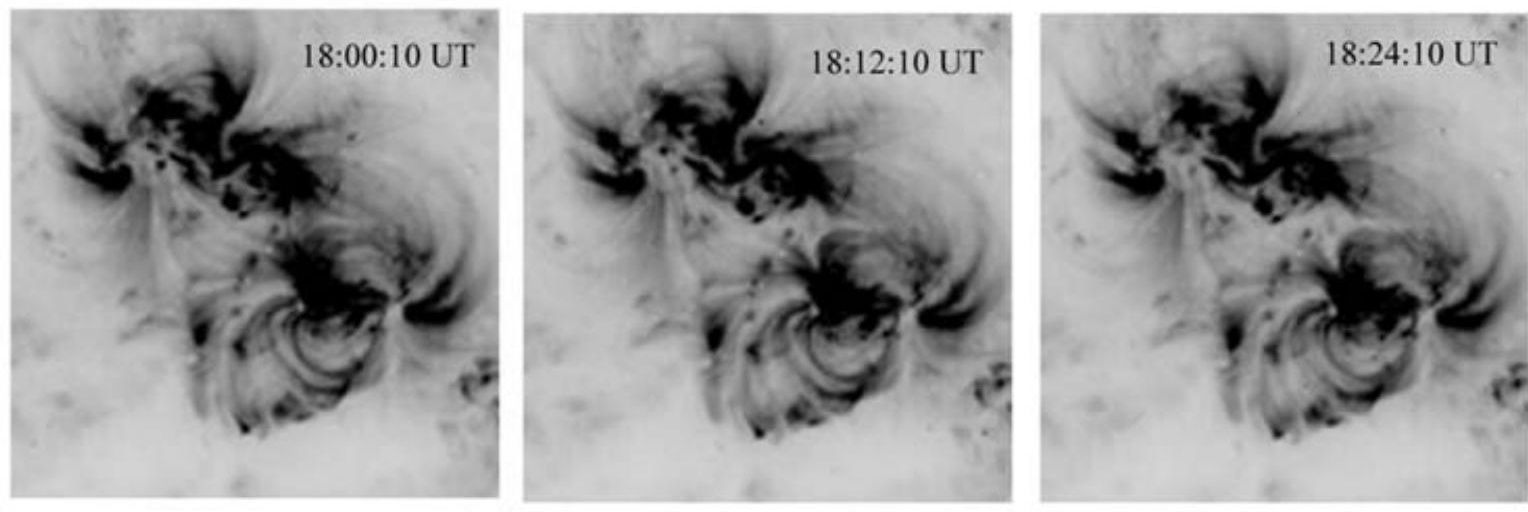

Figure 3. A series of SOHO EIT $195 \AA$ images showing the temporal evolution of the EUV emission from AR0321, AR0323 and the trans-equatorial region between them. Here, the gray-scale levels of the image have been inverted to provide better clarity.
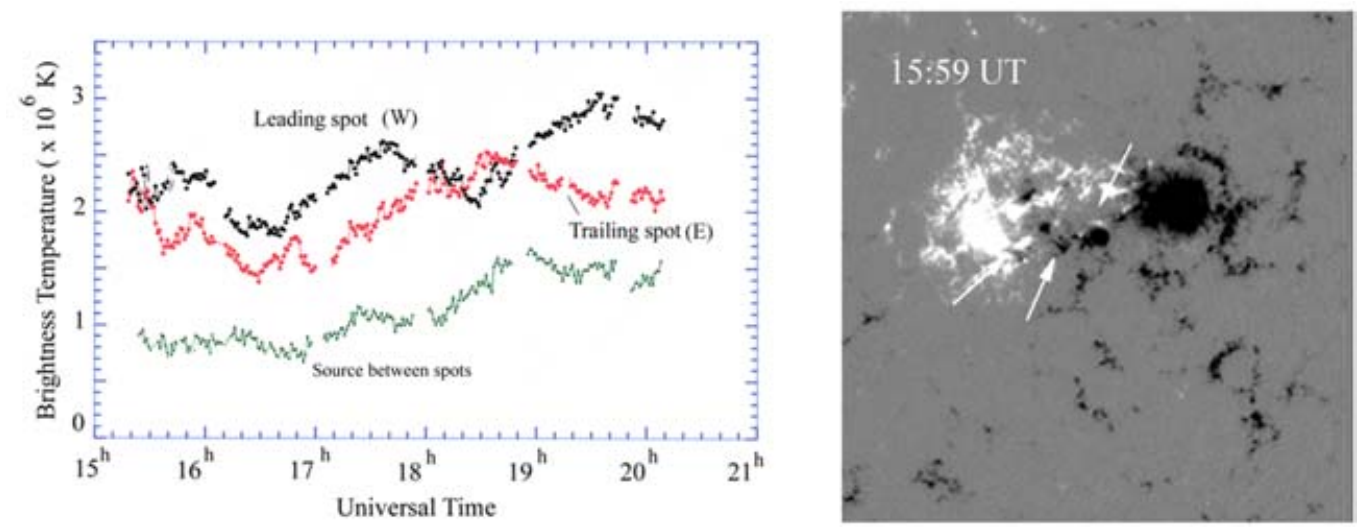

Figure 4. (left) Time profiles of the peak brightness temperature at $6 \mathrm{~cm}$ wavelength from the two sunspot-associated components within AR0323 and the source between them. (right) A series of MDI magnetograms of AR0323 at the times indicated. Here, the field-of-view is 4' x 4'. The arrows mark the location of emerging and evolving patches of magnetic flux that may be related to the evolving 6 and $20 \mathrm{~cm}$ emission from this region.

reconnection site at the equator, leading to the formation of the trans-equatorial loops themselves. These trans-equatorial loops may then act as conduits for particles that can propagate from one hemisphere to the other, possibly triggering burst activity in the remote footpoint region.

\section{Acknowledgements}

Solar observations at Tufts University are supported by NASA grant NAG5-12844.

\section{References}

Lang, K.R., Willson R.F., \& Trottet 1988, Astron. \& Astrophys. 199, 325.

Uchida, Y. et al 1992, Pub. Astron. Soc. Japan 44. L55.

Willson, R.F., Lang, K.R., \& Gary, D. 1993 Ap.J. 418, 490. 